\title{
Hybrid CPU and GPU Computation to Detect Lung Nodule in Computed Tomography Images
}

\author{
I Wayan Budi Sentana ${ }^{1}$, Naser Jawas ${ }^{2}$, Sri Andriati Asri ${ }^{3}$, \\ and Anggun Esti Wardani ${ }^{3}$ \\ ${ }^{1}$ Department of Information System, Bali State Polytechnic, \\ Jalan Raya Bukit Jimbaran, Badung, Indonesia, \\ ${ }^{2}$ Department of Computer System, STMIK STIKOM Bali, \\ Jalan Raya Puputan No. 86, Renon, Denpasar, Indonesia \\ ${ }^{3}$ Radiology Unit, Airlangga university Hospital, \\ Jalan Mayjen Dr. Moestopo No. 6-8, Surabaya, Indonesia
}

\begin{abstract}
Lung Nodule is a white patch on the thorax medical image, usually used as an early marker of lung cancer. This research aims to produce algorithms that can detect lung nodules automatically in CT images, by utilizing a combination of hybrid computing between Central Processing Unit (CPU) and Graphical Processing Unit (GPU). The framework used is Compute Unified Device Architecture, which consists of platform and programming model. The algorithm consists of several steps; read DICOM and data normalization, lung segmentation, candidate nodule extraction, and classification. Normalization is required to facilitate calculation by changing the data type ui16 to ui8. Furthermore, segmentation is used to separate the lung parts with other organs, where at this stage the Otsu Algorithm and Moore Neighborhood Tracing (MNT) are used. The next step is Lung Nodule Extraction, which aims to find the nodule candidate. The last step is a classification that utilizes the Support Vector Machine (SVM) to distinguish which one is nodule or not. The algorithm successfully detects near round nodules that are free-standing or not attached to other parts of organs. After undergoing ground truth tests, it was found that under some conditions, the algorithm has not been able to distinguish nodules and other strokes that resemble nodules. While in terms of computing speed is found a very surprising result because overall single CPU computing provides better results compared to hybrid CPU and GPU computing. Multiple morphology and transmission time to GPU contributed to the double execution time of hybrid model compared to single CPU. Adjustment in dataset grouping by detecting the nodule simultaneously for several dataset will also improve the performance of hybrid CPU and GPU computation.
\end{abstract}

Keywords: Lung Nodule, Hybrid Computing, GPU and CPU, CT images

\section{Introduction}

Pulmonary Nodule or better known as Lung Nodule is an identifier of certain lung disease including lung cancer[1]. It appears as a white -patches of lumps seen in the thoracic medical images. According to [2],[3], lung cancer become the deadliest diseases that caused 1.2-1.56 million deaths annually. The number is estimated to reach 17 million deaths by 2030[4]. According to [5], 80\% of this kind of cancer cases are detected after the patient experienced the disease with middle or upper stage. Hence, existence of automatic lung nodule application will have a significant impact along the way to handle the disease.

Radiologist have various techniques to detect nodule such as by utilized X-ray imaging, assisting computed tomography technique or known as CT scan and engaging Magnetic Resonance Imager (MRI) [4][6]. X-ray image is commonly used in the early screening stage of the nodule appearances, while CT scan and MRI is use to get the precise location as well as the size of nodules [1]. Although MRI provide more detailed imaging results, it has a drawback since its expensive price and fair performance on the soft tissue organ such in lung. Therefore,

Received: October $18^{\text {th }}, 2017$. Accepted: September $23^{\text {rd }}, 2018$

DOI: $10.15676 /$ ijeei.2018.10.3.4 
there so many radiologists using CT scans as diagnostic reference and so forth for computer scientist to conduct digital imaging research.

The existence of Application that can detect lung nodule automatically will help radiologist in their daily activities. According to [2], in average, a radiologist spent around 15-20 minutes to detect nodule per patient. Moreover, each radiologist performs forty-five exhausting examinations every day. Therefore, many researches working with image processing techniques to detect lung nodule automatically. Some of them are research conducted by [7], where the image segmentation process is done only in the parenchyma to find the solitary nodule by utilizing Gaussian mixture and hessian matrix, and using SVM for its classification. In addition [8] performs detection of solitary lung nodule by utilizing thresholding and SVM classification. As well as other research with different techniques and approaches.

Those researches only utilize the Central Processing Unit (CPU) to perform computing in image processing. None of these studies have used Graphical Processing Unit (GPU) to detect lung nodule in Computed Tomography Images. Previous researches related to GPU utilization for computing have been done by [9] to create computational simulations that utilize genetic algorithms. The research mentioned that its computation speeds up to 100 times the speed of single CPU usage. While the utilization of GPU for image processing has been done by [10], to classify hyperspectral data remote sensing results by using Support Vector Machine (SVM). The results showed an increase in speeds that reached 18.5 times the speed of single CPU data on 200 bands, 13 classes and 95,597 training data. Other studies related to the use of GPU in image processing in the form of a review of the suitability of GPU utilization in classification of MRI image results, conducted by [11] and [12]. Both studies claim that the GPU has the ability and the potential of the better in computing the classification of medical images.

Hence, this research proposes an application prototype to detect the lung nodule on the CT scan images by utilizing parallel computing of CPU and GPU. It is expected that the application can provide the same accuracy as previous research and have better computing speed.

\section{Algorithm Steps}

This research utilizes Compute Unified Device Architecture (CUDA) framework, which allows high-level languages to access the GPU kernel. CUDA consists of platforms and programming models by accommodating GPU engines that work parallel for all purposes, not just for graphical rendering purposes[13]. The fundamental difference between GPU and CPU is, GPU is designed as a device for intensive computing, while CPU is designed as an optimizer for flow control [9]. GPU uses many transistors for Arithmetic and Logic Unit (ALU), while many CPUs use it for control and data caching. This is why GPUs have better computational capabilities than CPUs, although the system still needs a CPU to manage the sharing of resources. So far, the process that requires the most resources ole GPU is graphical rendering. If not used for the process then GPU resources will not be optimally utilized.

Figure 1 shows the general steps that exist in this algorithm, while red-marked steps shows the parts of the algorithm optimized using the Array GPU. Not all parts of the algorithm are optimized with the GPU, since the limitations of existing libraries in the high-level programming language used. The steps of the algorithm are performed to all of slices and organized as follows:

\section{A. Read DICOM and Normalized Array Data}

DICOM is a standard data format in medical imagery as well in Computed Tomography (CT) images. CT images can consist of hundreds or even thousands of slices that are interconnected each other, so as if it can form a three-dimensional image. The first step in this algorithm is the process to read DICOM and normalize it, to make it lighter in the computation process. Normalization is done by changing the default data format DICOM in the form of Unsigned Integer (uint) 16 which has a range of values 0 to 65,535 , into uint 8 which has a range of values 0 to 255 . 


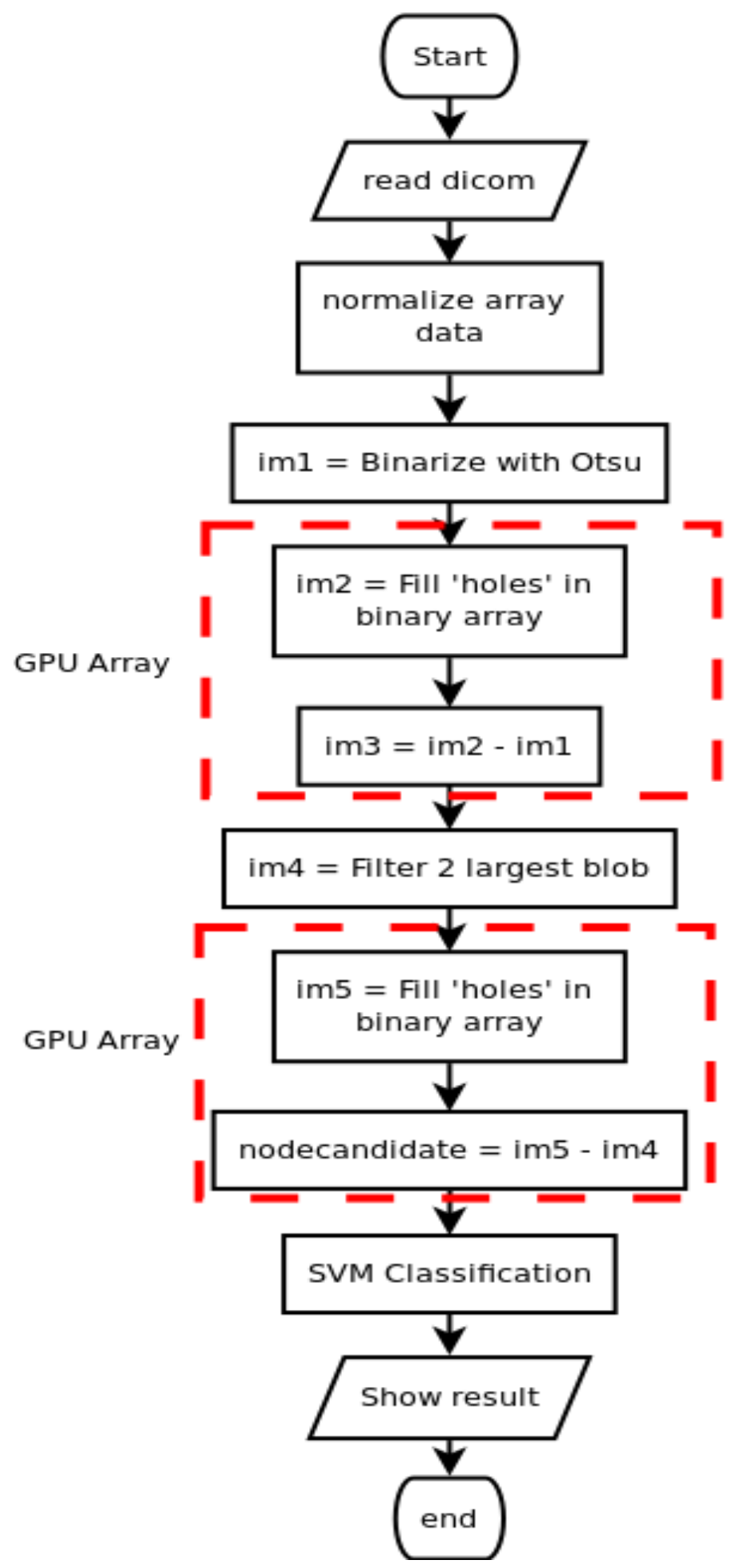

Figure 1. Optimized CPU-GPU Algorithm 


\section{B. Lung Segmentation}

The next step is to perform lung segmentation, where this step aims to separate the lung parts with other organs recorded in CT images. Segmentation consists of several stages, namely binarization with Otsu algorithm, filling parts of the lung with black or white with MNT algorithm, and determination of the largest blob that will form the image of the lung. Binarization used as an early step to separate the lung organ and another part of the images by contrasting the darker and brighter part in the images. In this case, Otsu algorithm seems to be the most proven to conduct this kind of operation.

For the certain purposes, pixel in given image represented in $\mathrm{L}$ gray level $(1,2,3 \ldots ., L)$. Let make $n_{i}$ denote the pixel number at level $i$ and $N$ denote the pixel total number, $N=\sum_{i}^{L} n_{i}$. The occurrence probability of $i$ is given by $p i=n_{i} / N$. Assume an image divided into two classes $C_{0}$ and $C_{l}$ using threshold $T$. $C_{0}$ contained pixels with level $[1, \ldots, T]$ and $C_{l}$ contained pixels with level $[T+1, \ldots, L]$. Assume $P_{O}(T)$ and $P_{l}(T)$ denoted the cumulative probabilities, $\mu_{0}(T)$ and $\mu_{1}(T)$ denote the mean level, and, $\sigma_{0}^{2}(T)$ and $\sigma_{1}^{2}(T)$ denote the variances of the $\mathrm{C} 0$ and $\mathrm{C} 1$ classes, respectively. This value is given by following:

$$
\begin{aligned}
& P_{0}(T)=\sum_{i=1}^{T} p_{i} \\
& P_{1}(T)=\sum_{i=T+1}^{L} p_{i}=1-P_{0}(T) \\
& \mu_{0}(T)=\sum_{i=1}^{T} i \frac{p_{i}}{P_{0}(T)}=\frac{1}{P_{0}(T)} \sum_{i=1}^{T} i p_{i} \\
& \mu_{1}(T)=\sum_{i=T+1}^{L} i \frac{p_{i}}{P_{1}(T)}=\frac{1}{P_{1}(T)} \sum_{i=T+1}^{L} i p_{i} \\
& \sigma_{0}^{2}(T)=\sum_{i=1}^{T}\left(i-\mu_{0}(T)\right)^{2} \frac{p_{i}}{p_{0}(T)} \\
& \sigma_{1}^{2}(T)=\sum_{i=T+1}^{L}\left(i-\mu_{1}(T)\right)^{2} \frac{p_{i}}{p_{1}(T)}
\end{aligned}
$$

Assume $\sigma_{b}^{2}(T), \sigma_{w}^{2}(T)$ and $\mu$ represent between-class variance, the within-class variance, and the mean level of the image:

$$
\begin{aligned}
& \mu=\sum_{i=1}^{L} i p_{i}=P_{0}(T) \mu_{0}(T)+P_{1}(T) \mu_{1}(T) \\
& \sigma_{b}^{2}(T)=P_{0}(T)\left(\mu_{0}(T)-\mu\right)^{2}+P_{1}(T)\left(\mu_{1}(T)-\mu\right)^{2} \\
& \sigma_{w}^{2}(T)=P_{0}(T) \sigma_{0}^{2}(T)+P_{1}(T) \sigma_{1}^{2}(T)
\end{aligned}
$$

The threshold calculated by maximizing the between-class variance proposed in Otsu that present as follow:

$$
T^{*}=\underset{1 \leq T<L}{\arg \max }\left\{\sigma_{b}^{2}(T)\right\}
$$

Maximizing the between-class variance is equal to minimizing the within-class variance criterion: 


$$
T^{*}=\underset{1 \leq T<L}{\arg } \min \left\{\sigma_{w}^{2}(T)\right\}
$$

Furthermore, the above threshold is same as the threshold calculated by maximizing the ratio between-class variance to within-class variance [14][15].

After the contrast generated by Otsu is clear, the next step is to fill the part of lung, located in the center of the image, by dark color based on the threshold formed by Otsu. This process is often called morphology. Moore Neighborhood Tracing (MNT) algorithm is tailoring to assist this process. MNT is pixel-following based algorithm that traces contour pixels in a predefined manner and then saves their coordinates in memory according to the trace order. This algorithm finds the next contour pixel using eight connected chain codes with a clockwise sequence starting from the rear pixel of the tracer, i.e., the tracer first moves toward the rear $\left(T\left(P_{\text {Rear }}, d_{\text {Rear }}\right)\right)$ and finds the next clockwise contour pixel, such as the left-rear, left, font-left, front, front-right, right and rear-right pixels[16].

\section{Candidate Nodule Extraction}

Once the lung area is determined, the next step is to determine the white part that will be the nodule candidate. This step uses the MNT algorithm to fill in the white color on the part that will be the nodule candidate. Giving color is intended to white areas can be perfectly closed, so that part is easily separated with other lung areas. After the nodule candidate is determined the next step is to subtract the pulmonary array with an array of nodules that will generate the nodule candidate areas.

\section{SVM Classification}

SVM then use to identify the nodule candidates defined in the previous step and then decide whether the candidate are nodules or not. It is done to separate between nodule and other part of lung. As its nature, SVM consist of training and testing stages. The basic concept of SVM is creating the decision planes that become a boundary of certain classes. The line is uses to isolate the object that have different class memberships. In this case, SVM using linear separable hyperplane that can separate the instances into two categories which is nodule and non-nodule. In the case when the objects cannot separate by linear hyperplane, SVM will transmute the instance into high-dimensional feature space that can be assisted by nonlinear transformation. SVM employs an iterative training algorithm to construct an optimal hyperplane. This process needed to find the minimum error function. SVM classification has general formula that can be described as followed [17][18][8]:

$$
\begin{aligned}
& \min \Phi(w)=\frac{1}{2}\|w\|^{2}+C \sum_{i=1}^{N} \xi_{i} \\
& \text { s.t } y_{i}\left(w^{T} . \phi\left(x_{i}\right)+b\right) \geq 1-\xi_{i},(i=1,2,3, \ldots N)
\end{aligned}
$$

in that formula, $\mathrm{C}$ represent parameter of penalty, meanwhile $\xi_{i}$ is use as parameter to handle linearly inseparable data. The goal is to minimize the object function $\Phi(w)$ which is shown in formula (12). That process is equal to maximize the gap or distance between two classes as well as to minimize the rate of misclassification. Variable $C$ needed to control the trade-off between the size of the margin and the slack variable penalty. The index $i$ use to label the $N$ training cases, $x_{i}$ represents the independent variables and $y_{i} \in \pm 1$ represents the class labels. Meanwhile, $\phi$ is kernel function used to transform data from the input to the feature space. For this case, RBF kernel function is used as appears in formula (13).

$$
k\left(x_{i}, x_{j}\right)=\exp \left(-\gamma\left\|x_{i}-x_{j}\right\|^{2}\right)
$$

$\gamma$ is the proportional inverse of kernel width. SVM classification has a common value for $\mathrm{C}$ and $\gamma$ to solved classification problems. 


\section{Results and Discussion}

Machine utilized to implement this algorithm is equipped with Intel Pentium Core i7 Processors up to $3.58 \mathrm{~Hz}, 8$ Giga Byte RAM, 1 Terra Byte Hard Drive and CUDA-enabled NVIDIA 940MX GPU Compute capability 5.0. This algorithm is implemented using MATLAB and the data used taken from "Keagle Repository Dataset". Near round solitary lung nodule with 20-pixels size successfully detected although its has drawback on detecting wall attaching nodule.

The result of each steps within the algorithm is shown in Figure 2 to Figure 6. Figure 2 shows the images after it converted into UI8 format. Visually, it has a big shape on the left-hand side of the Pulmonary, attached lung channel, that supposed to detected as nodule.

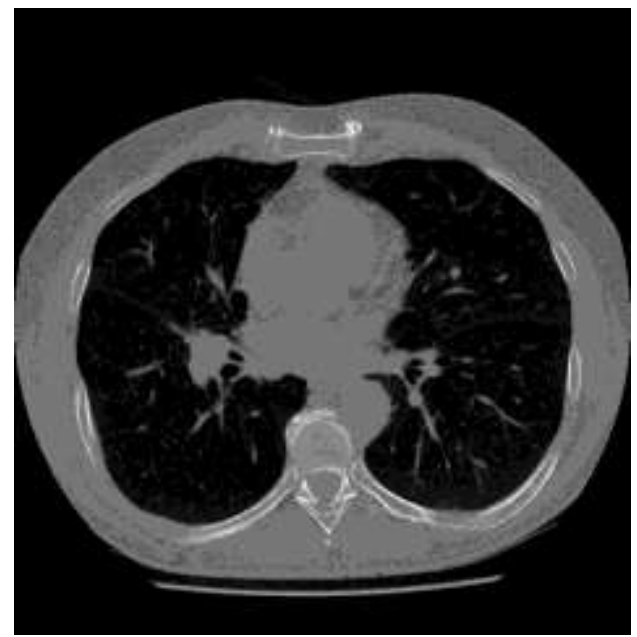

Figure 2. Result by Normalization

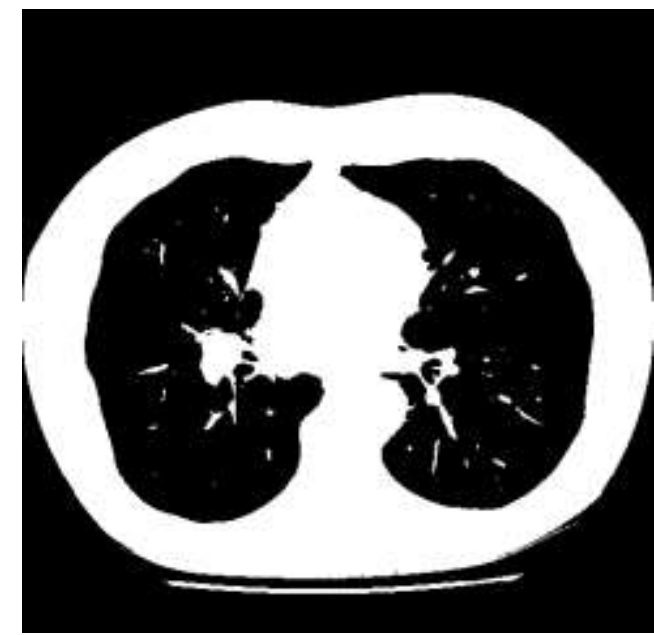

Figure 3. Result after Image Binarization using Otsu Algorithm

After the normalization process, Figure 3 shows the image after Otsu algorithm is tailored to binarized the image. This step caused the image became more contrast and it was needed to determine the threshold of the lung organ boundary, so the lung organ will be separated from the dark background image. It is found that the nodule located on the left part of the Pulmonary, which is part of Lung, excluded by the algorithm and detected as another soft tissue organ since it has different color gradation. 
MNT algorithm then tailored to segment the images and the result shown in Figure 4. In this step, the lung boundary is already identified according to the threshold determined by Otsu algorithm on the previous step. After the lung area was identified, Otsu algorithm is tailored again to separate the lung and the nodule as shown in Figure 5. The step produces white blob that become nodule candidate. Those candidates then classified to determine whether the candidate can be detected as nodule or vessel.

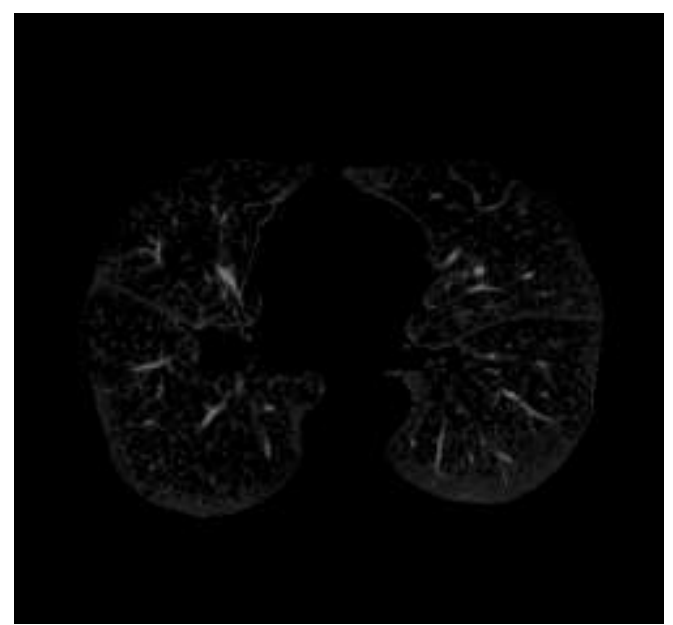

Figure 4. the result given by Segmentation step

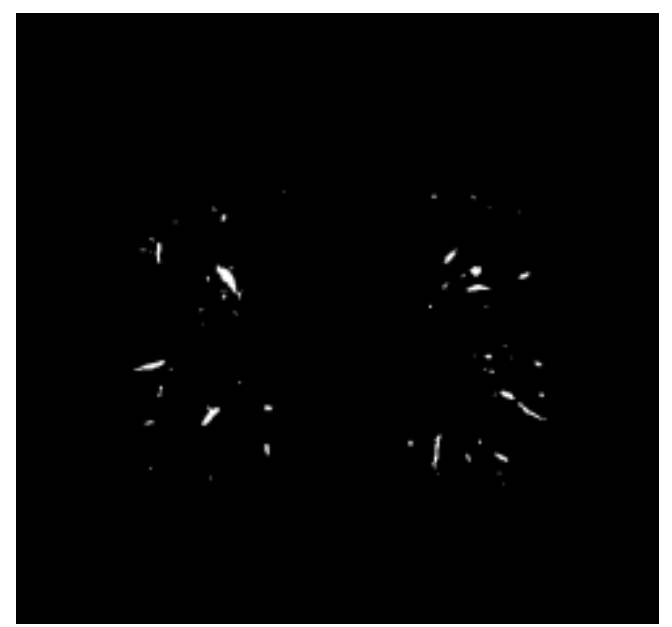

Figure 5. The result given by Nodule Candidate Extraction steps

After the nodule candidate successfully found, the next step is utilizing SVM to classify whether the candidates identified are nodule or not. As shown in Figure 6, the application marked the circle shape identified as nodule, that have size 20 pixel or above. The threshold of those pixel-size is manually setup. It is also shown the drawback of the algorithm since it cannot detect the extra size nodule on left pulmonary attached to lung channel or better known as bronchus. 


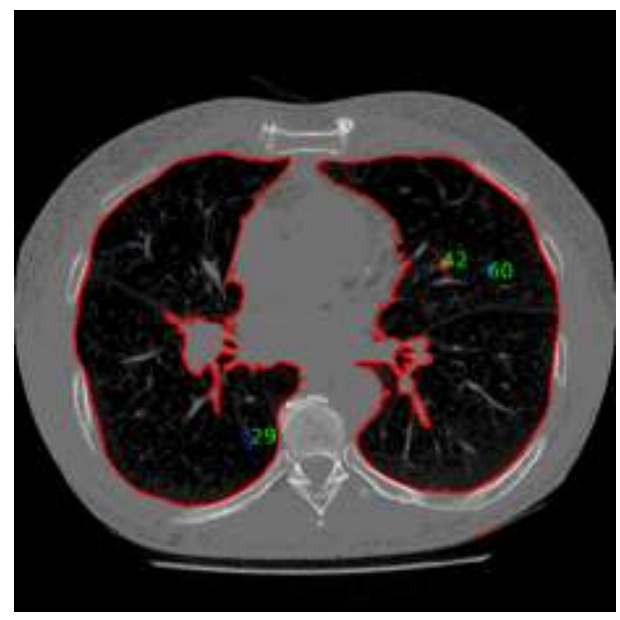

Figure 6. Results by SVM classification. Nodule position is combined with original images.

Application can detect some round tiny nodule.

After involving radiologist from Tabanan Regency General Hospital and Airlangga University Hospital, the algorithm found to have a drawback in detecting non-round nodule. Some advice also given to detect that kind of nodule as well as the anomaly that commonly appear along with it. To increase the accuracy, some enhancement on data pre-processing is needed in the future research, especially to detect Wall or Chanel attached lung nodule. In addition, it is also necessary to note the linkage between one slice and the other slices in the CT image, so the result of nodule detection is not only done per slice, which will be the cause of error detection.

In addition to the accuracy of detection, testing is done against the computing speed between single CPU and Hybrid CPU-GPU. The results are quite surprising because single CPUs provide better speed in computing, which is not the case in previous studies using the CUDA framework. The results of computational speed testing can be seen as in Table 1. Computational time increases when the number of slices CT image more and more.

Table 1. Computing Comparation between Single CPU and Hybrid GPU-CPU

\begin{tabular}{|cccc|}
\hline Patient ID & Slice \# & CPU Time (second) & CPU + GPU Time (Second) \\
\hline 5 & 146 & 1.6 & 3.4 \\
8 & 134 & 1.4 & 3.2 \\
11 & 217 & 2.4 & 5.3 \\
22 & 149 & 1.6 & 3.6 \\
35 & 155 & 1.7 & 3.8 \\
\hline
\end{tabular}

More details about the comparison of time consumption on each function are shown by Table 2. Computational speed comparison is only done in segmentation stage involving Array GPU only, considering that segmentation process takes the most time. In the table it appears that almost all functions running on the GPU array take longer time than the functions executed by a single CPU except in the "MinusArray" function.

The most significant difference is seen in the "MorphologyFilt" function, in which the execution time of the GPU-GPU hybrid reaches more than double the single CPU. This function is used to track and filling pixel by pixel arrays and does not require multithread array execution. This causes the CPU-GPU Hybrid execution to be the same as a single GPU but added with swapping time and caching time from CPU to GPU or vice versa, so the time will be doubled. 
Otherwise, different result found in the "MinusArray" function, which performs multithreading subtraction array operations that can be executed in parallel by the GPU. This is what causes the execution time of this function on hybrid CPU-GPU faster than single CPU. This action is subtraction of two images to separate the pulmonary area and its background image. The matrix can be parallelly transmitted and executed in GPU.

Table 2. Most Time Consuming Function

\begin{tabular}{|c|c|c|c|c|c|}
\hline $\begin{array}{l}\text { Patient } \\
\text { ID }\end{array}$ & CPU Time & GPU + CPU Time & $\begin{array}{l}\text { Patient } \\
\text { ID }\end{array}$ & CPU Time & GPU + CPU Time \\
\hline \multirow[t]{6}{*}{5} & $\begin{array}{l}\text { MorphologyFill } \\
(0.55 \mathrm{~s})\end{array}$ & $\begin{array}{l}\text { MorphologyFill } \\
(1.52 \mathrm{~s})\end{array}$ & \multirow[t]{6}{*}{22} & $\begin{array}{l}\text { MorphologyFill } \\
(0.64 \mathrm{~s})\end{array}$ & $\begin{array}{l}\text { MorphologyFill } \\
(1.56 \mathrm{~s})\end{array}$ \\
\hline & $\begin{array}{l}\text { BwAreaFilt } \\
(0.35 \mathrm{~s})\end{array}$ & $\begin{array}{l}\text { GpuCpuArray } \\
(0.36 \mathrm{~s})\end{array}$ & & $\begin{array}{l}\text { BwAreaFilt } \\
(0.36 \mathrm{~s})\end{array}$ & $\begin{array}{l}\text { GpuCpuArray } \\
(0.36 \mathrm{~s})\end{array}$ \\
\hline & $\begin{array}{l}\text { GreyThresh } \\
(0.14 \mathrm{~s})\end{array}$ & BwAreaFilt (0.36s) & & $\begin{array}{l}\text { GreyThresh } \\
(0.19 \mathrm{~s})\end{array}$ & BwAreaFilt (0.31s) \\
\hline & \multirow[t]{3}{*}{$\begin{array}{l}\text { MinusArray } \\
(0.14 \mathrm{~s})\end{array}$} & GreyThresh (0.21s) & & $\begin{array}{l}\text { MinusArray } \\
(0.14 \mathrm{~s})\end{array}$ & GreyThresh $(0.27 \mathrm{~s})$ \\
\hline & & MinusArray (0.04s) & & & MinusArray (0.07s) \\
\hline & & CpuGpuArray $(0.05 \mathrm{~s})$ & & & CpuGpuArray(0.06s) \\
\hline \multirow[t]{6}{*}{8} & $\begin{array}{l}\text { MorphologyFill } \\
(0.58 \mathrm{~s})\end{array}$ & $\begin{array}{l}\text { MorphologyFill } \\
(1.36 \mathrm{~s})\end{array}$ & \multirow[t]{6}{*}{35} & $\begin{array}{l}\text { MorphologyFill } \\
(0.62 \mathrm{~s})\end{array}$ & $\begin{array}{l}\text { MorphologyFill } \\
(1.53 \mathrm{~s})\end{array}$ \\
\hline & $\begin{array}{l}\text { BwAreaFilt } \\
(0.34 \mathrm{~s})\end{array}$ & $\begin{array}{l}\text { GpuCpuArray } \\
(0.27 \mathrm{~s})\end{array}$ & & $\begin{array}{l}\text { BwAreaFilt } \\
(0.33 \mathrm{~s})\end{array}$ & $\begin{array}{l}\text { GpuCpuArray } \\
(0.32 \mathrm{~s})\end{array}$ \\
\hline & $\begin{array}{l}\text { GreyThresh } \\
(0.15 \mathrm{~s})\end{array}$ & BwAreaFilt (0.35s) & & $\begin{array}{l}\text { GreyThresh } \\
(0.22 \mathrm{~s})\end{array}$ & BwAreaFilt (0.34s) \\
\hline & \multirow[t]{3}{*}{$\begin{array}{l}\text { MinusArray } \\
(0.17 \mathrm{~s})\end{array}$} & GreyThresh $(0.23 \mathrm{~s})$ & & $\begin{array}{l}\text { MinusArray } \\
(0.13 \mathrm{~s})\end{array}$ & GreyThresh (0.22s) \\
\hline & & MinusArray (0.03s) & & & MinusArray (0.06s) \\
\hline & & CpuGpuArray $(0.05 \mathrm{~s})$ & & & CpuGpuArray(0.06s) \\
\hline \multirow[t]{6}{*}{11} & $\begin{array}{l}\text { MorphologyFill } \\
(0.89 \mathrm{~s})\end{array}$ & $\begin{array}{l}\text { MorphologyFill } \\
(1.56 \mathrm{~s})\end{array}$ & & & \\
\hline & $\begin{array}{l}\text { BwAreaFilt } \\
(0.38 \mathrm{~s})\end{array}$ & $\begin{array}{l}\text { GpuCpuArray } \\
(0.29 \mathrm{~s})\end{array}$ & & & \\
\hline & $\begin{array}{l}\text { GreyThresh } \\
(0.31 \mathrm{~s})\end{array}$ & BwAreaFilt (0.37s) & & & \\
\hline & \multirow[t]{3}{*}{$\begin{array}{l}\text { MinusArray } \\
(0.19 \mathrm{~s})\end{array}$} & GreyThresh $(0.29 \mathrm{~s})$ & & & \\
\hline & & MinusArray (0.10s) & & & \\
\hline & & CpuGpuArray(0.08s) & & & \\
\hline
\end{tabular}


I Wayan Budi Sentana, et al.

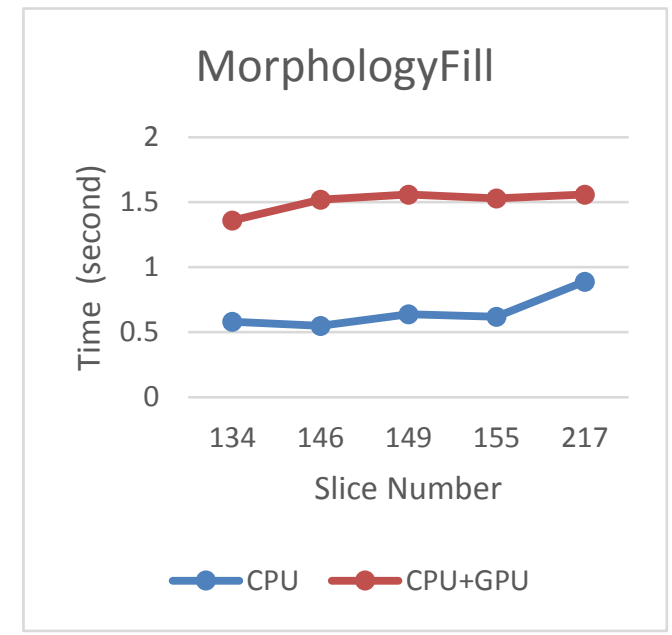

Figure 7.a. Morphological Process Comparation

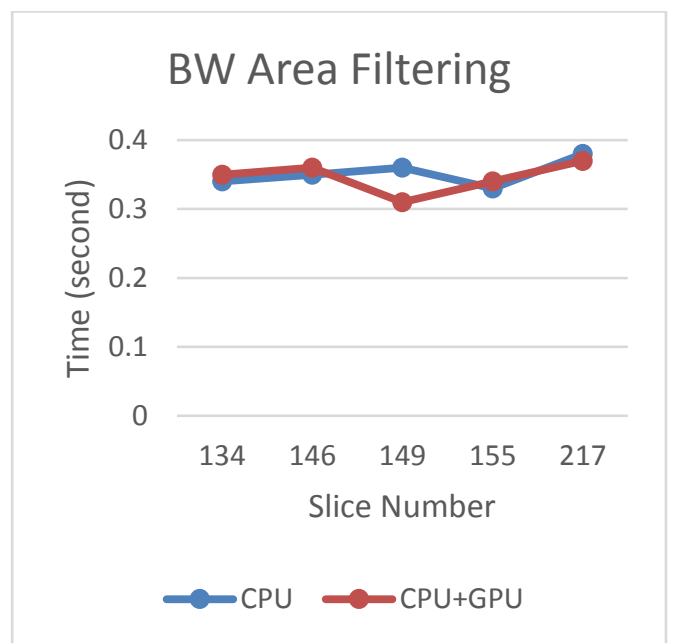

Figure 7.b. Black and White Area Filtering

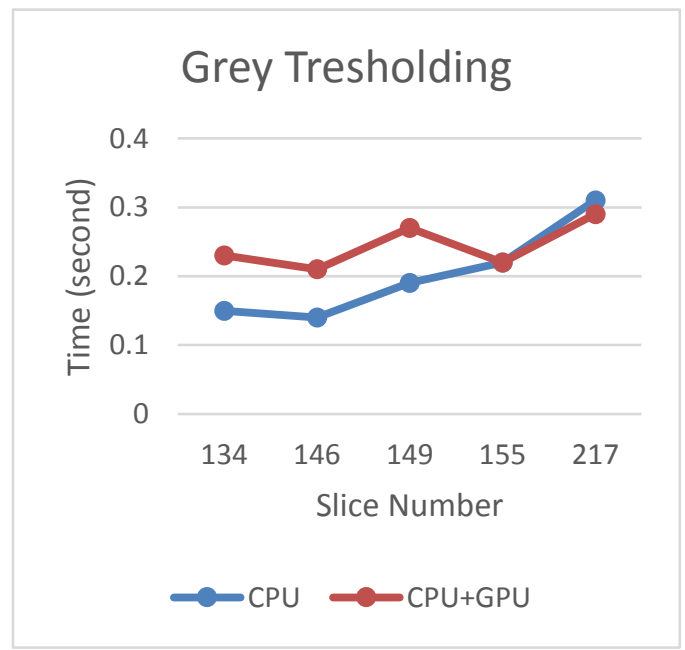

Figure 7.c. Grey Thresholding Comparation 


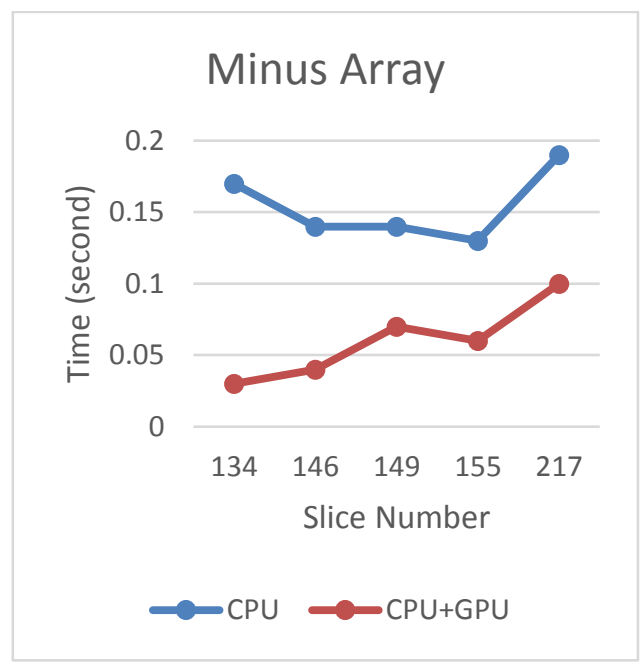

Figure 7.d. Minus Array comparation

The following graphs in Figure 7.a to 7.d show the results of computational comparisons between single CPU and hybrid CPU-GPU for several processes in the lung nodule detection. Vertical axis shows the amount of consumption time in seconds and the horizontal axis is the number of slices on the CT scan image. Morphological process in Figure 7.a shows that the typical of this process is not compatible to carry out in hybrid environment since the computing result of hybrid CPU and GPU show its inferiority compare to single CPU. Morphology is the technique of processing digital images using shapes as guidelines in processing. The value of each pixel in the digital image results obtained through the process of comparison between the corresponding pixels in the input digital image with the neighbor's pixel. Morphology depends on the appearance order of the pixel and not to the numeric value of the pixel, so that the morphology technique is appropriate when used to perform binary image and grayscale image processing. Morphology consist of erosion and dilation process. Dilation is the process of adding pixels to the boundary of an object in the digital image input, while erosion is the process of removing / reducing pixels at the boundary of an object. The number of pixels added or removed from the object boundary on the digital image input depends on the size and shape of the structuring element used. In this case, Morphology use to fill the background color surrounded by homogeneous foreground colors. That kind of process is done serially by checking pixel by pixel of an image. Hence, the computing time will be longer since the process is equal as it done by a single CPU plus the time to transmit the data to the GPU.

Meanwhile, the Black and White area filtering and Grey Thresholding as shown in Figure 7.b and 7.c, figure out that computing result are straight correlated to the slice number in CT scan images. On a certain number of image slices, hybrid CPU and GPU model shows a better computation results and increased continuously when the slices number is getting high. Black and White filtering and Grey Thresholding are light process that does not need large computation resources. So, when the slices number to be executed is in small amount, the time spent to transmit data to GPU is much larger then the execution time in its owned CPU. Hence, the hybrid model resulting a higher computation time compared to single CPU. But when the slices amount is getting high, the GPU will show that it is perform better than single CPU as its nature to compute.

The real performance of GPU shown by Figure 7.d, which are Minus Array process. This proces conduct multithreading array subtraction simultaneously. The result shows that hybrid model give much faster computation than single CPU. In this process, GPU can parallelly conduct computation so its perform better than single CPU. However, the overall computation time of hybrid model is higher since it need a process to transmit data from CPU to GPU. Moreover, in this prototype, the lung nodule detection process was performed for each set of CT 
scan images that group by each patient. The performance of hybrid model can perform better for Black and White Process and Grey Thresholding if the computation conduct for several set of images simultaneously.

\section{Conclusions and Future Research}

The application has successfully detecting almost round nodule within more than 20-pixels. Although it can detect certain nodule, the algorithm has some drawback since it cannot detect wall and channel attached nodule. Segmentation step need to have some enhancement, so it can improve the result on classification. In the other hand, overall computation speed of Single CPU is better than hybrid CPU-GPU proposed in this research. Multiple morphological process seems to be more appropriate to conduct in single CPU because it causes the computation time almost double in hybrid model since the additional time to transmit data to GPU. The time use to compute serially and time for data to travel along the bus are contributed for the computation time. Another worth noting is the way to group the dataset. The detection process should be conduct simultaneously for several image datasets, so the GPU execution time will outperform the transmission time.

\section{Acknowledgement}

This research funded by Directorate General for Research strengthening and Development, Ministry of Research, Technology and Higher Education, Republic of Indonesia through the scheme of prime university research grants. We are also really appreciate the radiological assistance from Tabanan General Hospital and Airlangga University Hospital which has provide time for consultation and discussion.

\section{References}

[1]. N. Mesanovic, M. Grgic, H. Huseinagic, M. Males, E. Skejic, and M. Smajlovic, "Automatic CT Image Segmentation of the Lungs with Region Growing Algorithm," Int. J. Eng. Adv. Technol., vol. 4, no. 5, pp. 395-400, 2015.

[2]. H. M. Orozco, S. Member, O. Osiris, V. Villegas, S. Member, and H. De Jes, "Lung Nodule Classification in CT Thorax Images using Support Vector Machines," in 12th Mexican International Conference on Artificial Intelligence, 2013, pp. 277-282.

[3]. P. Bhuvaneswari and A. B. Therese, "Detection of Cancer in Lung With K-NN Classification Using Genetic Algorithm," Procedia Mater. Sci., vol. 10, no. Cnt 2014, pp. 433-440, 2015.

[4]. K. Mya, M. Tun, and A. S. Khaing, "Feature Extraction and Classification of Lung," Int. J. Eng. Res. Technol., vol. 3, no. 3, pp. 2204-2210, 2014.

[5]. F. V. Farahani, A. Ahmadi, and M. H. F. Zarandi, "Lung Nodule Diagnosis from CT Images Based on Ensemble Learning," 2015.

[6]. C. Bhuvaneswari, P. Aruna, and D. Loganathan, "Classification of Lung Diseases by Image Processing Techniques Using Computed Tomography Images," Int. J. Adv. Comput. Res., vol. 4, no. 1, pp. 2277-7970, 2014.

[7]. A. M. Santos, A. O. De Carvalho Filho, A. C. Silva, A. C. De Paiva, R. A. Nunes, and M. Gattass, "Automatic detection of small lung nodules in 3D CT data using Gaussian mixture models, Tsallis entropy and SVM," Eng. Appl. Artif. Intell., vol. 36, pp. 27-39, 2014.

[8]. P. Eskandarian and J. Bagherzadeh, "Computer-Aided Detection of Pulmonary Nodules based on SVM in Thoracic CT Images," in 7th International Conference on Information and Knowledge Technology, 2015, pp. 0-5.

[9]. M. C. Lau and R. Srinivasan, "A hybrid CPU-Graphics Processing Unit (GPU) approach for computationally efficient simulation-optimization," Comput. Chem. Eng., vol. 87, pp. 49-62, 2016.

[10]. K. Tan, J. Zhang, Q. Du, S. Member, and X. Wang, "GPU Parallel Implementation of Support Vector Machines for Hyperspectral Image Classification," IEEE J. Sel. Top. Appl. Earth Obs. Remote Sens., vol. 1, pp. 1-10, 2015. 
[11]. E. Smistad, T. L. Falch, M. Bozorgi, A. C. Elster, and F. Lindseth, "Medical image segmentation on GPUs - A comprehensive review.," Med. Image Anal., vol. 20, no. 1, pp. 1-18, 2014.

[12]. A. Eklund, P. Dufort, D. Forsberg, and S. M. Laconte, "Medical image processing on the GPU - Past , present and future," Med. Image Anal., vol. 17, no. 8, pp. 1073-1094, 2013.

[13]. NVIDIA, Cuda c programming guide, no. September. USA, 2015.

[14]. X. Xu, S. Xu, L. Jin, and E. Song, "Characteristic analysis of Otsu threshold and its applications," Pattern Recognit. Lett., vol. 32, no. 7, pp. 956-961, 2011.

[15]. C. Sha, J. Hou, and H. Cui, "A Robust 2D Otsu' s Thresholding Method in Image Segmentation," J. Vis. Commun. Image Represent., no. October, 2016.

[16]. J. Seo, S. Chae, J. Shim, D. Kim, C. Cheong, and T. Han, "Fast Contour-Tracing Algorithm Based on a Pixel-Following Method for Image Sensors," MDPI J. sensors, vol. 16, no. 353, pp. 1-27, 2016.

[17]. P. Chen, L. Yuan, Y. He, and S. Luo, "An improved SVM classifier based on double application in analogue circuit diagnosis Reference : To appear in : Neurocomputing," Neurocomputing, 2015.

[18]. H. R. Tizhoosh and F. Khalvati, "Medical Image Classification via SVM using LBP Features from Saliency-Based Folded Data," pp. 0-4, 2015.
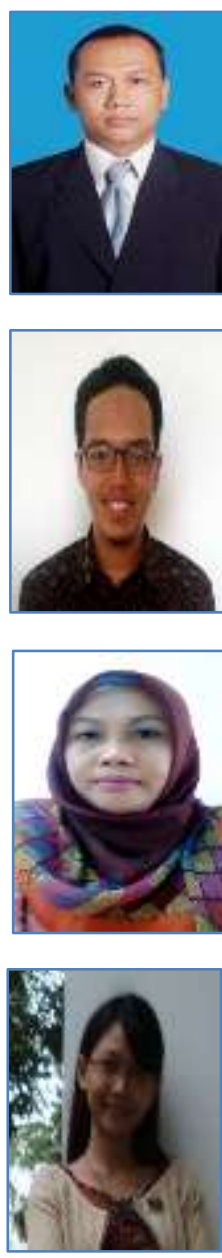

Anggun Esti Wardani, obtained a Bachelor of Medicine (S.Ked) and a doctorate profession (dr.), From Airlangga University Surabaya, respectively in 2005 and 2008. Obtained a degree of Radiology Specialist (Sp. Rad.) From Specialist Education Program Doctor I, Faculty of Medicine Airlangga University Surabaya, in 2012. Lecturer at the Faculty of Medicine, University of Airlangga and also radiologist at Airlangga University Hospital Surabaya. Currently taking Doctor II Specialist Education Program (sub-specialist) at the Faculty of Medicine, Airlangga University. Anggun Esti Wardani can be reached at Campus C Universitas Airlangga Surabaya or via angioanggun@gmail.com. 